\title{
Flexible Grouping for Enhanced Energy Utilization Efficiency in Battery Energy Storage Systems
}

\author{
Weiping Diao ${ }^{1,2}$, Jiuchun Jiang ${ }^{1,2}$, Hui Liang ${ }^{1,2}$, Caiping Zhang ${ }^{1,2, *}$, Yan Jiang ${ }^{1,2}$, Leyi Wang ${ }^{3}$ \\ and Biqiang $\mathrm{Mu}^{4}$ \\ 1 National Active Distribution Network Technology Research Center (NANTEC), Beijing Jiaotong University, \\ Beijing 100044, China; wpdiao@bjtu.edu.cn (W.D.); jcjiang@bjtu.edu.cn (J.J.); hliang@bjtu.edu.cn (H.L.); \\ 14121418@bjtu.edu.cn (Y.J.) \\ 2 Collaborative Innovation Center of Electric Vehicles in Beijing, Beijing Jiaotong University, \\ Beijing 100044, China \\ 3 Department of Electrical and Computer Engineering, Wayne State University, Detroit, MI 48202, USA; \\ lywang@wayne.edu \\ 4 The Key Laboratory of Systems and Control of CAS, Institute of Systems Science, Academy of Mathematics \\ and Systems Science, Chinese Academy of Sciences, Beijing 100190, China; bqmu@amss.ac.cn \\ * Correspondence: zhangcaiping@bjtu.edu.cn; Tel.: +86-135-8171-1177
}

Academic Editor: Sheng S. Zhang

Received: 3 May 2016; Accepted: 24 June 2016; Published: 29 June 2016

\begin{abstract}
As a critical subsystem in electric vehicles and smart grids, a battery energy storage system plays an essential role in enhancement of reliable operation and system performance. In such applications, a battery energy storage system is required to provide high energy utilization efficiency, as well as reliability. However, capacity inconsistency of batteries affects energy utilization efficiency dramatically; and the situation becomes more severe after hundreds of cycles because battery capacities change randomly due to non-uniform aging. Capacity mismatch can be solved by decomposing a cluster of batteries in series into several low voltage battery packs. This paper introduces a new analysis method to optimize energy utilization efficiency by finding the best number of batteries in a pack, based on capacity distribution, order statistics, central limit theorem, and converter efficiency. Considering both battery energy utilization and power electronics efficiency, it establishes that there is a maximum energy utilization efficiency under a given capacity distribution among a certain number of batteries, which provides a basic analysis for system-level optimization of a battery system throughout its life cycle. Quantitative analysis results based on aging data are illustrated, and a prototype of flexible energy storage systems is built to verify this analysis.
\end{abstract}

Keywords: battery; flexible group; converter efficiency; energy utilization efficiency

\section{Introduction}

With fast-growing distributed generation (DG) in recent years, smart grids have evolved into a comprehensive networked system composed of renewable resources, energy storage systems, and loads. An energy storage system (ESS) plays an important role in smart grids and is required to offer high energy / power density, long life cycle, fast response, and a mature industry chain. Battery energy storage systems (BESS) have been applied to many demonstration projects because of their reasonable energy density and cost. The cost of a BESS depends on its original price, lifetime, and energy utilization efficiency. This paper is focused on analyzing energy utilization efficiency.

In conventional BESS, hundreds of batteries are connected in series to attain a certain level of voltage, which requires high consistency among batteries. However, due to different conditions during the process of manufacture and utilization, the system always suffers from batteries' inconsistency [1]. The battery pack's available energy is determined by the capacities, relative state of charge (SOC) levels 
and the terminal voltages of all the batteries. In the original stage, most batteries are highly consistent, which means that most capacity and energy of each battery in the pack can be utilized. However, after hundreds of cycles, capacity distribution of batteries will show a divergent trend. In such cases, some batteries cannot be fully charged/discharged, and the energy utilization efficiency will decrease a lot.

Figure 1a shows capacity differences in a pack, Figure $1 \mathrm{~b}-\mathrm{d}$ shows that SOC differences further aggravate the inconsistency level. As shown in Figure 1a, when the minimum battery is the first one to be fully charged and discharged, the actual capacity of the pack is the minimum battery's capacity. Batteries in this pack do not need to be balanced, but the capacity is limited to the minimum battery. Otherwise, if the minimum battery isn't the first one to be fully charged or discharged due to SOC differences as shown in Figure $1 b-d$, actual available capacity is even less than the minimum capacity of all the batteries in a pack. Passive equalization of the battery pack can solve the problem of SOC differences but has no effect on capacity differences [2].

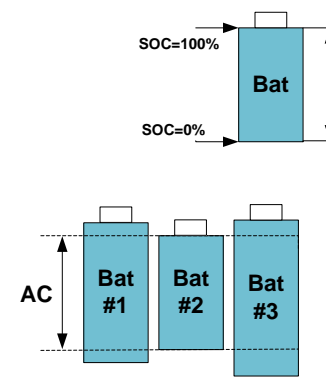

(a)

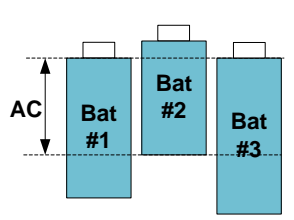

(c)
AC:

Actual

Capacity

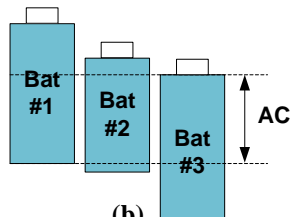

(b)

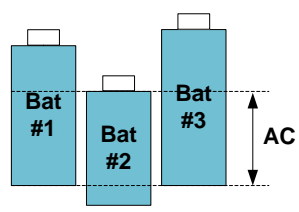

(d)

Figure 1. Batteries' relative SOC in one pack: (a) Battery 2 is the first one to be fully charged and discharged; (b) Battery 3 is the first one to be fully charged, Battery 1 is the first one to be fully discharged; (c) Battery 1,3 are the first ones to be fully charged, Battery 2 is the first one to be fully discharged; (d) Battery 2 is the first one to be fully charged, Battery 1,3 are the first ones to be fully discharged.

A cluster of $N$ batteries serially connected can be divided into $n$ low-voltage battery packs through power electronics [3]. In this way, a conventional group of batteries connected with a high-power converter is shifted to multiple flexible groups. A flexible group integrates several batteries and a low-power converter into a module as shown in Figure 2. The power converter can be a DC/DC or a DC / AC converter to realize individual power control of each low-voltage battery pack. A certain number of modules can be cascaded or parallel-connected to a DC bus or AC bus.

In this case, high consistency is only required inside each low-voltage battery pack with $N / n$ batteries (assume that all groups have the same size). It is not required in the total number of batteries. This integration unit is pluggable and expandable with high fault redundancy. The notion of integration of battery and power electronics has been presented in [1]. High compatibility and reliability make this module viable and advantageous [4,5]. A multilevel converter is a good choice to implement the flexible group, by replacing flying capacitors or isolated dc sources by battery packs.

Using additional power electronics introduces additional cost/area/weight. However, this cost will be offset by the extended lifetime of batteries and increased energy utilization efficiency over the life cycle. Modular design of each low-voltage converter further reduces the cost of power electronics, which is similar to PV systems where micro-inverters tend to replace centralized inverters. Therefore, 
with the continuous decreasing cost of power electronic devices, the proposed design will make more sense in the future. Moreover, with flexible groups, the reliability and maintainability are enhanced, further reducing running and maintenance cost. In typical applications of large-scale battery systems such as smart grids, the volume and weight are not very critical factors.

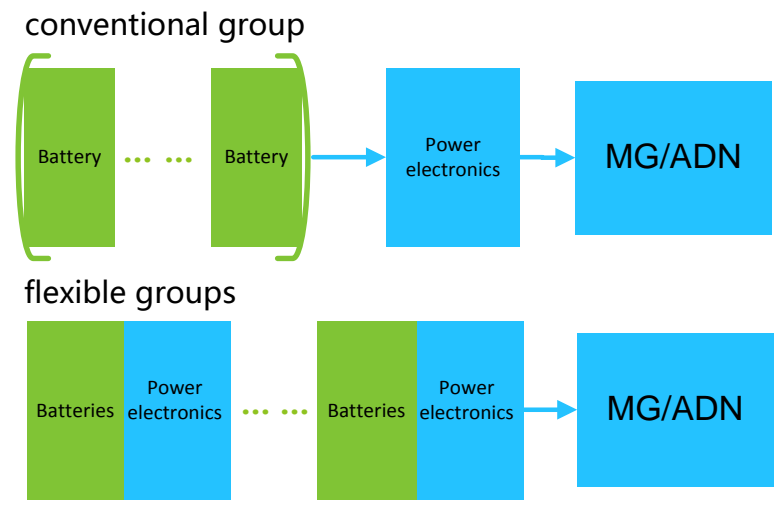

Figure 2. The diagram of a conventional group and flexible groups.

Many studies have been conducted on flexible groups. To improve efficiency, some topologies and control methods have been proposed. References [6-10] showed five different topologies with control methods respectively, of which the H-bridge converter is most commonly used [7] and topologies proposed in [8-10] could reduce the number of switches and loss. To guarantee the continuous operation, fault tolerant control was studied in [11] to enhance the reliability. Another important direction in the BESS is optimization control for batteries [6,7,12-15]. These control methods, such as SOC balancing among different modules, provided a useful power allocation algorithm during operation.

It is imperative to verify the feasibility of flexible groups from the perspective of efficiency, reliability, and cost of the system, which was investigated in [16]. Loss of Thevenin's equivalent resistance was taken into account in calculating efficiency of batteries; nevertheless, it did not address the impact of capacity inconsistency. Reference [17] calculated the reliability of different single phase battery-to-grid converter interfacing schemes. It found that second-life batteries were the least reliable part of the system. Thus, minimizing the number of batteries in a pack was considered a key part of increasing system reliability of a single unit. This conclusion further supported the significance of dividing batteries into groups in this paper. However, the question that how many batteries in one pack is the most cost-effective and reliable was not discussed. Similarly in [5], the original battery pack was divided into two packs, which enhanced lifetime and safety significantly. However, there was only one case studied in the paper, and the relationship between energy utilization efficiency and the number of batteries in one pack was still not clear.

To study this relationship, experimental and operational data are needed. It was demonstrated that statistical data showed approximately identical properties for the same usage of batteries although each battery's regression is random [18]. This study provided a theoretical support to calculate the energy utilization efficiency of BESS. A large amount of data from new and aged lithium-ion batteries was analyzed for mathematical fitting of capacity and impedance distribution in [19].

Based on the statistical characteristics of batteries' data, calculation of energy utilization efficiency after a certain number of batteries are divided into several groups is feasible in this paper.

As is shown in Figure 2, energy utilization of the module is determined by not only available energy of the battery pack $\left(E_{n}\right)$ but also the converter efficiency $(\eta)$, which leads to the total energy $E_{n} \times \eta$. If the number of groups $(n)$ among $N$ batteries increases, $E_{n}$ will go up but $\eta$ will decrease. Under a certain capacity distribution, there is an optimal range of $n$ (number of groups) to obtain maximum energy utilization efficiency. 
This paper is organized as follows. Section 2 introduces the Thevenin model and the derivation process of energy utilization calculation of batteries in various cases where the number of groups and capacity distribution are different. Section 3 calculates converter efficiency of different numbers of groups as described in Section 2. Section 4 combines these two factors and establishes a model to work out the optimal number of groups under different capacity distributions. Section 5 presents two experiments to demonstrate flexible groups. The energy utilization efficiency of each configuration is measured and compared in the first experiment, and individual control is realized in the BESS prototype proposed. Finally, Section 6 concludes the paper with the main findings and the potential future research directions.

\section{Analysis of Energy Utilization of a Battery Pack}

\subsection{Capacity Analysis Based on a Battery Model}

A typical battery model is shown in Figure 3. $U_{O C V}$ is the open circuit voltage, $U_{R}$ and $U_{P}$ are ohmic voltage drop and polarization voltage, respectively. $U_{O}$ (terminal voltage) reaches the cut-off voltage ahead of $U_{O C V}$ because of $U_{R}$ and $U_{P}$, which means that the battery will not be fully charged/discharged. However, this problem can be solved through changing current to rather small (e.g., $0.1 \mathrm{C}$ ) at the end of charge/discharge; thus, the voltage drop $U_{R}$ and $U_{P}$ can be neglected and the battery can approximately be regarded as fully charged/discharged. In this case, depth of discharge (DOD) is approximately $100 \%$ and the maximum available capacity of the battery can be fully used $[2,20]$.

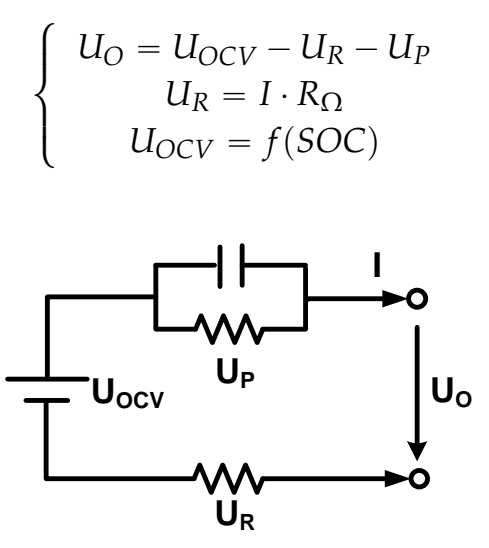

Figure 3. Thevinen model.

\subsection{Energy Utilization Analysis}

Denote the maximum capacity of the $N$ batteries by $\left\{Q_{i}, i=1, \ldots, N\right\}$, which is defined by the protection circuit's voltage thresholds during charge and discharge. Assume that $\left\{Q_{i}, i=1, \ldots, N\right\}$ are independent and identically distributed(i.i.d.) normal random variables with mean $\mu$ and variance $\sigma^{2}$. Thus, the density function $f(y)$ is:

$$
f(y)=\frac{1}{\sqrt{2 \pi} \sigma} \exp \left(-\frac{(t-\mu)^{2}}{2 \sigma^{2}}\right)
$$

The distribution function $F(y)$ can be obtained. Since SOC inconsistency of batteries in a pack can be solved by balancing, only situations as in Figure 1a are calculated. The actual capacity for the pack is the minimum capacity of all the batteries. When $N$ batteries are connected in series, the actual capacity of the pack is:

$$
Q_{N}=\min _{1 \leqslant i \leqslant N} Q_{i}
$$

The actual energy of the $N$ batteries connected in series is [2]: 


$$
E_{N}=\min _{1 \leqslant i \leqslant N} Q_{i} \cdot \sum_{i=1}^{N} U_{m i}
$$

where $U_{m i}$ is the mid-value voltage of battery $i$. The mid-value voltage is generally measured when SOC is $50 \%$. Divide the $N$ batteries into $n$ groups, the number of batteries per group is $N / n$. Thus, the capacity of the $j$ th group that can be utilized is:

$$
Q_{j}=\min _{1 \leqslant i \leqslant N / n} Q_{(j-1) / n+i}
$$

The total energy of the $n$ battery packs is:

$$
\begin{aligned}
E_{n} & =\sum_{j=1}^{n}\left[\min _{1 \leqslant i \leqslant N / n} Q_{(j-1) / n+i} \cdot \sum_{i=1}^{N / n} U_{m[(j-1) / n+i]}\right] \\
& =\sum_{j=1}^{n}\left[\min _{1 \leqslant i \leqslant N / n} Q_{(j-1) / n+i} \cdot \frac{N}{n} U_{a v j}\right]
\end{aligned}
$$

where $U_{a v j}$ is the average mid-value voltage $\left(U_{m}\right)$ of batteries in the $j$ th group and index " $m$ " means medium-value. It is known that among the large number of battery samples, if more batteries are connected in series, the average voltage of the batteries is closer to average level of samples. Since these batteries are selected from batteries with same distribution, if the number of batteries in one pack reaches a certain value, $U_{a v j}$ is considered the same for each pack. This analysis is a one-dimension random variable calculation. Thus:

$$
E_{n}=U_{a v} \cdot \frac{N}{n} \sum_{j=1}^{n}\left[\min _{1 \leqslant i \leqslant N / n} Q_{(j-1) / n+i}\right]
$$

Define:

$$
Y_{j}=\min _{1 \leqslant i \leqslant N / n} Q_{(j-1) / n+i}
$$

Thus:

$$
E_{n}=U_{a v} \cdot \frac{N}{n} \sum_{j=1}^{n} Y_{j}
$$

Define:

$$
Q_{s u m}=\frac{N}{n} \sum_{j=1}^{n} Y_{j}
$$

Therefore:

$$
E_{n}=U_{a v} \cdot Q_{\text {sum }}
$$

where $\left\{Y_{j}, j=1, \ldots, n\right\}$ is a sequence of i.i.d. random variables. The distribution function of $Y_{j}$ is [21]:

$$
\begin{aligned}
F_{Y}(y)=P\left(Y_{1} \leqslant y\right) & =P\left(\min _{1 \leqslant i \leqslant N / n} Q_{N(j-1) / n+i} \leqslant y\right)=P\left(\bigcup_{j=1}^{N / n} Q_{j} \leqslant y\right) \\
& =1-P\left(\bigcap_{j=1}^{N / n} Q_{j}>y\right)=1-\prod_{j=1}^{N / n}(1-F(y)) \\
& =1-(1-F(y))^{N / n}
\end{aligned}
$$

Accordingly, the density function of $Y_{j}$ is:

$$
f_{Y}(y)=F^{\prime} Y(y)=N(1-F(y))^{N / n-1} f(y) / n
$$


Since $f(y)$ and $F(y)$ are known, $f_{Y}(y)$ is available. The expectation and variance of $Y_{j}$ are denoted by $E Y_{j}$ and $\operatorname{Var}\left(Y_{j}\right)$. Assume that there is a lower threshold $T_{n}$ with probability $\alpha$ :

$$
\frac{N}{n} \sum_{j=1}^{n} Y_{j} \geqslant T_{n}
$$

That is:

$$
P\left(\frac{N}{n} \sum_{j=1}^{n} Y_{j} \geqslant T_{n}\right) \geqslant \alpha
$$

This results in:

$$
P\left(\sum_{j=1}^{n} Y_{j} \geqslant n T_{n} / N\right) \geqslant \alpha
$$

Based on the Central Limit Theorem [22]:

$$
\frac{\sum_{j=1}^{n}\left(Y_{j}-E Y_{j}\right)}{\sqrt{n \operatorname{Var}\left(Y_{j}\right)}} \stackrel{n \rightarrow \infty}{\longrightarrow} N(0,1)
$$

Thus, we have:

$$
T_{n} \leqslant N \phi^{-1}(1-\alpha) \sqrt{\frac{\operatorname{Var}\left(Y_{j}\right)}{n}}+N E Y_{j}
$$

with probability $\alpha$.

Figure 4 shows a group of data from $\mathrm{LiFePO}_{4}$ batteries which have been cycled for about 1500 times. The capacities have deteriorated from the original rated $60 \mathrm{Ah}$ to a distribution of mean 49.46 Ah and standard deviation 1.499 Ah after three years of service, which shows approximately Gaussian distribution characteristics. The energy utilization efficiency is analyzed based on these experimental data.

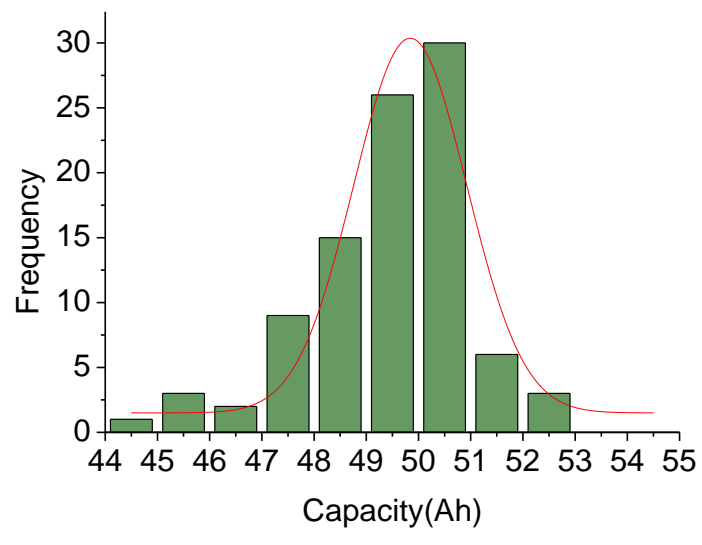

Figure 4. Battery capacity data.

Assume that there are $N=100$ batteries in total. All of the batteries are randomly grouped instead of deliberately screened. Figure 5 shows the relationship between $E_{n}$ and the number of groups $(n)$ where probability $\alpha$ is 0.95 and 0.99 , respectively, based on data mentioned in Section 2.1. Under given capacity distribution, $E_{n}$ rises with $n$ increasing as expected. However, when $n$ is larger than approximately five, the increasing speed of $E_{n}$ begins to go down. 


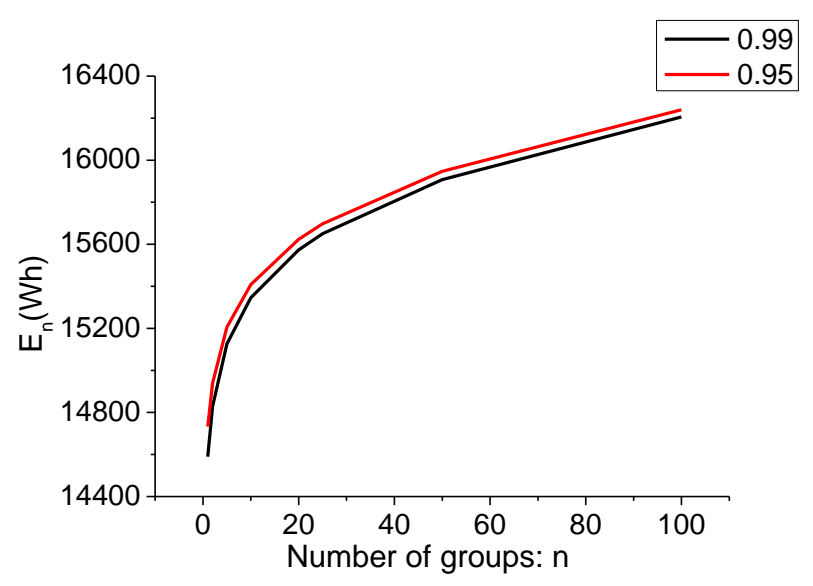

Figure 5. $E_{n}$ under given capacity distribution.

During the process of utilization, the mean of capacity and the inconsistency level are gradually changing. Reference [23] showed that the discrepancies among batteries were increasing with aging spread which had a major influence on the energy utilization as well as lifetime. Within the life cycle, assume that the mean of the capacity among all the batteries deteriorates from $100 \%$ to $80 \%$ of nominal value and the inconsistency level increases from 0 to $15 \%$ of capacity as shown in Table 1 . This changing process is taken as linear and the data still fits a Gaussian distribution. Figure 6 shows $E_{n}$ under different capacity inconsistency levels. From the slopes of these curves, it can be concluded that when the inconsistency level is higher, dividing the 100 batteries into more groups has a more significant impact on $E_{n}$.

Table 1. Mean and standard deviation in different stages.

\begin{tabular}{ccccccc}
\hline Cycle(Times) & $\mathbf{0}$ & $\mathbf{3 0 0}$ & $\mathbf{6 0 0}$ & $\mathbf{9 0 0}$ & $\mathbf{1 2 0 0}$ & $\mathbf{1 5 0 0}$ \\
\hline Mean $(\mathrm{Ah})$ & $60(100 \%)$ & $57.6(96 \%)$ & $55.2(92 \%)$ & $52.8(88 \%)$ & $50.4(84 \%)$ & $48(80 \%)$ \\
Standard deviation $(\mathrm{Ah})$ & $0(0 \%)$ & $0.36(3 \%)$ & $0.69(6 \%)$ & $0.99(9 \%)$ & $1.26(12 \%)$ & $1.5(15 \%)$ \\
\hline
\end{tabular}

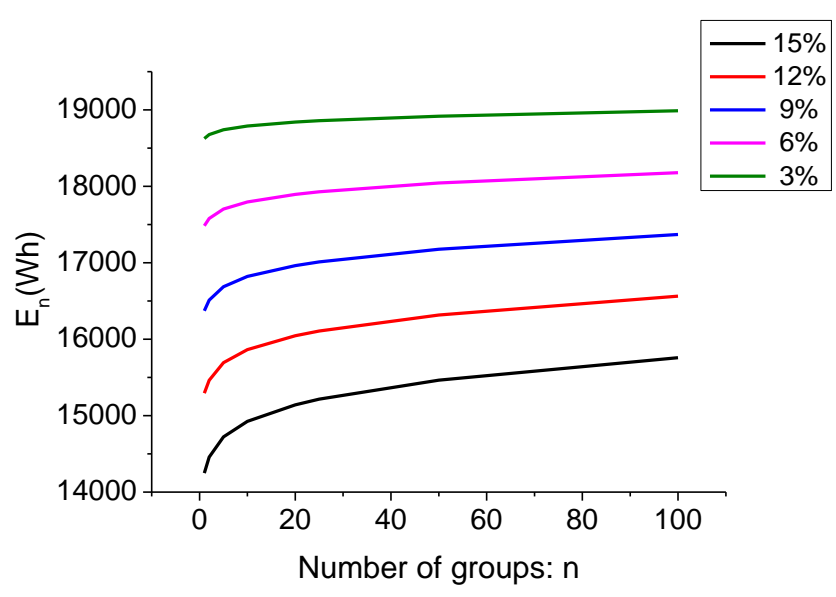

Figure 6. $E_{n}$ under different capacity inconsistency levels.

\section{Analysis of Converter Efficiency}

\subsection{Topology Illustration of Converter}

There are various multilevel converters that can be used for BESS, since they have lower $\mathrm{d} v / \mathrm{d} t$, lower switching frequency, lower rating of current and voltage, as well as lower distortion. H-bridge 
is the most common and easy-control application as shown in Figure 7 [7]. The number of conduction switches is $2 n$.

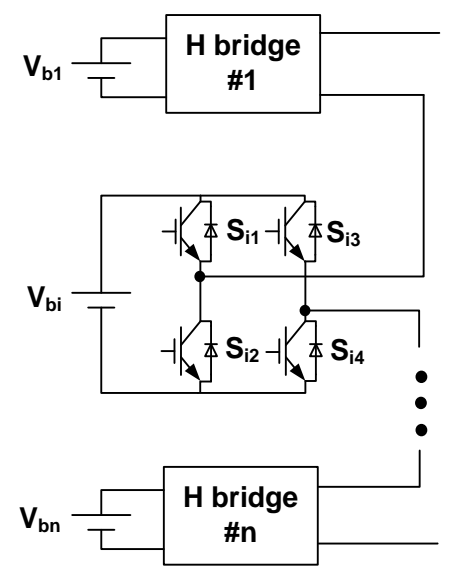

Figure 7. Cascaded H-bridge converter.

An H-bridge module can produce positive voltage, zero voltage, and negative voltage. The switches' state of different output voltages is shown in Table 2. By controlling the duty ratio of switches in each H-bridge, the converter can output the desired voltage and realize individual control of each H-bridge.

Table 2. Switches' state of different output voltages.

\begin{tabular}{ccccc}
\hline $\mathbf{U}_{\mathbf{H}}$ & $\mathbf{S}_{\mathbf{i} 1}$ & $\mathbf{S}_{\mathbf{i} 2}$ & $\mathbf{S}_{\mathbf{i} 3}$ & $\mathbf{S}_{\mathbf{i} 4}$ \\
\hline$+\mathrm{V}_{\mathrm{b}}$ & 1 & 0 & 0 & 1 \\
0 & 0 & 1 & 0 & 1 \\
0 & 1 & 0 & 1 & 0 \\
$\mathrm{~V}_{\mathrm{b}}$ & 0 & 1 & 1 & 0 \\
\hline
\end{tabular}

Some other topologies have been proposed with fewer switches to realize lower loss of semiconductor devices. The most popular one is the multilevel DC link inverter presented in [8] as shown in Figure 8. The number of conduction switches is $n+2$, which is less than H-bridge converter when $n>2$. Other topologies, such as in $[9,10]$, have $n+1$ and $1.5 n$ conduction switches, respectively. However, these two topologies are not modular enough to realize easy control. In this paper, the H-bridge converter is cited to calculate the efficiency.

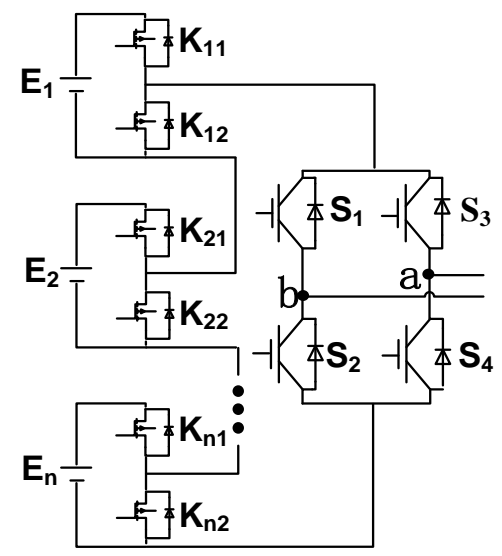

Figure 8. Multilevel DC link inverter. 


\subsection{Converter Efficiency Calculation}

Semiconductor loss is thought as the main cause of total loss. The calculation is comprised of loss in the MOSFETs and source-drain diodes. To calculate the average conduction loss of semiconductors, only a half cycle calculation is needed [24].

\subsubsection{Loss of MOSFET}

Reference [24] calculated the power losses of semiconductors in the three-phase inverter. The method is cited and some parameters, such as dynamic resistance of diodes and forward voltage drop of MOSFET, are neglected in this paper. The conduction loss of MOSFET is:

$$
P_{\text {cond } 1}=\left(\frac{1}{8}+\frac{1}{3 \pi} m \cos \phi\right) R_{D S(o n)} I_{p}^{2}
$$

where $I_{p}$ is the peak value of the current, $m$ is the modulation index, $\cos \Phi$ is the power factor. The switching loss of MOSFET is:

$$
P_{s w 1}=f \cdot \frac{1}{\pi} \cdot\left(W_{o n}+W_{o f f}\right) \frac{I_{r m s}}{I_{\text {rate }}} \cdot \frac{U_{r m s}}{U_{\text {rate }}}
$$

Thus the total loss of MOSFET is:

$$
P_{\text {loss } 1}=\left(\frac{1}{8}+\frac{1}{3 \pi} m \cos \phi\right) R_{D S(o n)} I_{p}^{2}+f \cdot \frac{1}{\pi} \cdot\left(W_{o n}+W_{\text {off }}\right) \frac{I_{r m s}}{I_{\text {rate }}} \cdot \frac{U_{\text {rms }}}{U_{\text {rate }}}
$$

\subsubsection{Loss of Diode}

The same case goes for the calculation of diode loss. Conduction loss of diode is:

$$
P_{\text {cond } 2}=\left(\frac{1}{2 \pi}-\frac{1}{8} m \cos \phi\right) V_{D S} I_{p}
$$

The switching loss of diode mainly comprises of reverse recovery loss (turn-off loss), which derives:

$$
P_{s w 2}=\frac{1}{2} V_{D S} I_{R M} t_{a} f+\frac{1}{4} V_{R M} I_{R M} t_{b} f
$$

Thus, the total loss of diode is:

$$
P_{\text {loss } 2}=\left(\frac{1}{2 \pi}-\frac{1}{8} m \cos \phi\right) V_{D S} I_{p}+\frac{1}{2} V_{D S} I_{R M} t_{a} f+\frac{1}{4} V_{R M} I_{R M} t_{b} f
$$

3.2.3. Total Loss

$$
P_{\text {loss }}=4 n\left(P_{\text {loss } 1}+P_{\text {loss } 2}\right)
$$

If the $N$ batteries are divided into $n$ groups, the voltage level of each battery pack $V_{C}$ is:

$$
V_{C}=N \cdot U_{O} / n
$$

where $U_{O}$ is the terminal voltage of the battery. Semiconductors' on-resistance is related to $V_{C}$. However, it does not show a linear relationship between them. For different numbers of groups, semiconductors are of different ratings, as shown in Table 3.

The nominal power of the system is:

$$
P=N \cdot U_{O} \cdot I=I_{r m s} \cdot U_{r m s}
$$


Thus:

$$
I_{r m s}=\frac{N \cdot U_{O} \cdot I}{U_{r m s}}
$$

where $I_{r m s}$ and $U_{r m s}$ are the rms current and voltage of grid side respectively, $I_{r m s}$ does not change with the number of groups $(n)$. The efficiency of power electronics $\eta$ is:

$$
\eta=1-\frac{P_{\text {loss }}}{N \cdot U_{O} \cdot I} \times 100 \%
$$

As mentioned in Section 2, there are 100 batteries in total. Discharging current is 2 C (100 A). According to Equation (28), RMS current of the AC side is 168 A. Module parameters and loss calculation are shown in Table 3. The efficiency with different numbers of groups is illustrated in Figure 9. As the number of groups goes up, converter efficiency decreases because there are more semiconductors, which cause more loss.

\begin{tabular}{|c|c|c|c|c|c|c|c|}
\hline \multirow{2}{*}{$\begin{array}{l}\text { Number of } \\
\text { Groups/n }\end{array}$} & \multicolumn{3}{|c|}{ Module Parameter } & \multicolumn{4}{|c|}{ Efficiency Analysis } \\
\hline & $\begin{array}{c}\text { Number of } \\
\text { Each Pack/N/n }\end{array}$ & $\begin{array}{l}\text { Module Voltage } \\
\text { (V) }\end{array}$ & $\begin{array}{l}\text { MOSFET } \\
\text { Parameter }\end{array}$ & $P_{\text {loss1 }}$ & $P_{\text {loss2 }}$ & $P_{\text {loss }}$ & $H$ \\
\hline 2 & 50 & $160-210$ & $500 \mathrm{~V} / 497 \mathrm{~A}$ & $113 \mathrm{~W}$ & $19 \mathrm{~W}$ & $1056 \mathrm{~W}$ & $97.146 \%$ \\
\hline 5 & 20 & $64-84$ & $200 \mathrm{~V} / 417 \mathrm{~A}$ & $50 \mathrm{~W}$ & $16 \mathrm{~W}$ & $1320 \mathrm{~W}$ & $96.432 \%$ \\
\hline 10 & 10 & $32-42$ & $100 \mathrm{~V} / 420 \mathrm{~A}$ & $34 \mathrm{~W}$ & $17 \mathrm{~W}$ & $2043 \mathrm{~W}$ & $94.479 \%$ \\
\hline 20 & 5 & $16-21$ & $75 \mathrm{~V} / 480 \mathrm{~A}$ & $23 \mathrm{~W}$ & $17 \mathrm{~W}$ & $3200 \mathrm{~W}$ & $91.35 \%$ \\
\hline 25 & 4 & $12.8-16.8$ & $75 \mathrm{~V} / 480 \mathrm{~A}$ & $23 \mathrm{~W}$ & $17 \mathrm{~W}$ & $4000 \mathrm{~W}$ & $89.189 \%$ \\
\hline 50 & 2 & $6.4-8.4$ & $75 \mathrm{~V} / 480 \mathrm{~A}$ & $22 \mathrm{~W}$ & $17 \mathrm{~W}$ & $7800 \mathrm{~W}$ & $78.919 \%$ \\
\hline
\end{tabular}

Table 3. Converter efficiency calculation.

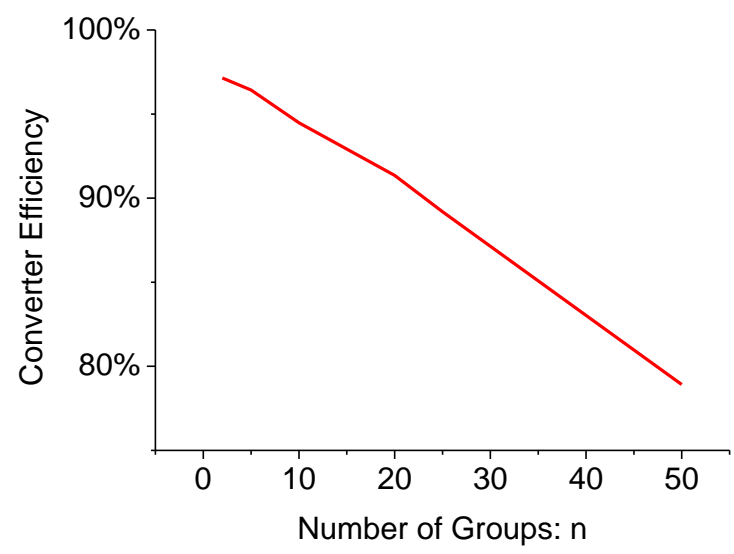

Figure 9. Relationship between $\eta$ and the number of groups.

\section{Energy Utilization Efficiency of Flexible Group Module}

Define $Q_{F}$

$$
Q_{F}=Q_{\text {sum }} \cdot \eta
$$

Combined with Equation (11), energy utilization of the module is:

$$
E=E_{n} \cdot \eta=U_{a v} \cdot Q_{F}
$$

Energy utilization efficiency $\eta_{E}$ is:

$$
\eta_{E}=\frac{E}{E_{b a t}} \times 100 \%
$$


where $E_{b a t}$ is the maximum energy that can be obtained from the $N$ batteries when they are used separately instead of connected in series.

From the analysis in Sections 2 and 3, it is observed that $Q_{\text {sum }}(n)$ is a monotone increasing function, while $\eta(n)$ is a monotone decreasing function. Thus, the curve shape of their product $Q_{F}(n)$ is close to a parabola. As shown in Equation (31) and (32), energy utilization efficiency is the maximum when $Q_{F}$ reaches the vertex because $U_{a v}$ and $E_{b a t}$ do not change with $n$; thus, the optimal number of groups is determined.

As shown in Figure 10, based on the retired battery data mentioned in Section 2.1 (with a mean value of $49.46 \mathrm{Ah}$ and standard deviation of $1.499 \mathrm{Ah}$ ) where probability $\alpha$ is 0.95 and 0.99 , respectively, $\eta_{E}$ is the maximum when the number of groups is five. Maximum energy utilization efficiency of this retired battery sample is approximately $90 \%$.

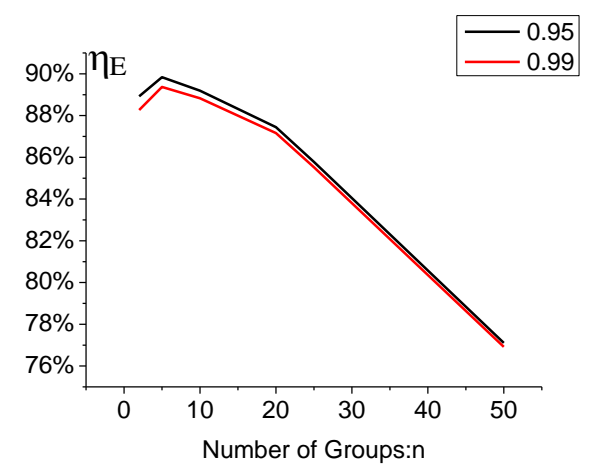

Figure 10. Relationship between $\eta_{E}$ and the number of groups.

A series of calculations to get maximum energy utilization efficiency have been done based on the analysis above. Table 4 shows the optimal number of groups in different aging stages. It can be seen that in different stages, the optimal number of groups is different. A higher level of inconsistency leads to a larger optimal number of groups. In the calculation, the optimal configuration depends on the number of cycles because that the capacity distribution changes with the number of cycles. Although the regression of batteries is random, the capacity distribution still fits statistical characteristics. Thus, the conditions of batteries after a certain number of cycles can be estimated and the optimal configuration is determined by the average energy utilization efficiency throughout the life cycle.

Table 4. Optimal number of groups in different stages.

\begin{tabular}{ccccccc}
\hline Cycle(Times) & $\mathbf{0}$ & $\mathbf{3 0 0}$ & $\mathbf{6 0 0}$ & $\mathbf{9 0 0}$ & $\mathbf{1 2 0 0}$ & $\mathbf{1 5 0 0}$ \\
\hline Mean $(\mathrm{Ah})$ & $100 \%(60)$ & $96 \%(57.6)$ & $92 \%(55.2)$ & $88 \%(52.8)$ & $84 \%(50.4)$ & $80 \%(48)$ \\
$\begin{array}{c}\text { Standard } \\
\text { deviation }(\mathrm{Ah})\end{array}$ & $0(0)$ & $3 \%(0.36)$ & $6 \%(0.69)$ & $9 \%(0.99)$ & $12 \%(1.26)$ & $15 \%(1.5)$ \\
$\begin{array}{c}\text { Optimal number } \\
\text { of groups }\end{array}$ & 1 & 2 & $2-5$ & 5 & 5 & 5 \\
\hline
\end{tabular}

In addition to increasing the energy utilization efficiency, multiple groups can lessen the speed of battery deterioration and increase the flexibility of operation. In this model proposed, the input variables are the number of total batteries $N$, battery capacity distribution characteristics, converter topology, charge/discharge current, and control frequency. The output variable is the optimal number of groups. It should be noted that the output variable could also be an optimal range, because the value $N / n$ must be an integral in the calculation. 


\section{Experiments}

To verify the analysis, two experiments are designed. The first one is to test the energy utilization efficiency of batteries with different groups as illustrated in Section 2. The second one is to verify the feasibility of flexible groups in BESS.

\subsection{Experiment 1}

24 batteries are selected and divided into different number of groups. The mean and standard deviation of their capacities are 49.27 Ah and 1.82 Ah, respectively. There are three configurations in this experiment. In the first and second configurations, the 24 batteries are divided into one and two groups, respectively. In the third configuration, the 24 batteries are divided into three groups, where there are two scenarios of grouping batteries. In the first scenario, the batteries are randomly divided into three groups. In the second scenario, batteries are sorted based on their capacities, and batteries with similar capacities are connected in a pack. In these configurations, the numbers of batteries in a pack are 24,12 , and eight, respectively.

These batteries are fully charged at the start. In the first configuration, the battery group is discharged to its low-voltage cut-off point, then charged to its high-voltage cut-off point, and finally discharged to its low-voltage cut-off point again. In the second and third configuration, the battery groups are firstly charged and then discharged to respective cut-off points. The total energy is measured for the last discharge process. The experiment is performed on an Arbin BT2000 (Arbin Instruments, College Station, USA). Results are shown in Table 5.

Table 5. Experiment 1 Results.

\begin{tabular}{|c|c|c|c|c|c|c|}
\hline Items & \multicolumn{2}{|c|}{$\begin{array}{c}\text { Discharge Capacity } \\
\text { (Ah) }\end{array}$} & $\begin{array}{c}\text { Discharge Energy } \\
\text { (Wh) }\end{array}$ & $\begin{array}{l}\text { Energy Utilization } \\
\text { Efficiency }\end{array}$ & $\begin{array}{l}\text { Theoretical Value } \\
\qquad(\alpha=0.95)\end{array}$ & Error \\
\hline Configuration 1 & \multicolumn{2}{|c|}{44.22} & 3444.084 & $89.74 \%$ & $89.39 \%$ & $0.35 \%$ \\
\hline \multirow{2}{*}{ Configuration 2} & G1 & 43.82 & 1691.860 & \multirow{2}{*}{$90.89 \%$} & \multirow{2}{*}{$91.29 \%$} & \multirow{2}{*}{$-0.4 \%$} \\
\hline & G2 & 46.14 & 1796.272 & & & \\
\hline \multirow{3}{*}{ Configuration 3.1} & G1 & 43.66 & 1123.480 & \multirow{3}{*}{$92.37 \%$} & \multirow{3}{*}{$92.38 \%$} & \multirow{3}{*}{$-0.01 \%$} \\
\hline & G2 & 45.87 & 1185.187 & & & \\
\hline & G3 & 47.85 & 1236.292 & & & \\
\hline \multirow{3}{*}{ Configuration 3.2} & G1 & 42.56 & 1102.626 & \multirow{3}{*}{$93.06 \%$} & \multirow{3}{*}{$92.38 \%$} & \multirow{3}{*}{$0.68 \%$} \\
\hline & G2 & 47.67 & 1230.209 & & & \\
\hline & G3 & 47.90 & 1238.653 & & & \\
\hline
\end{tabular}

From the data of random groups (Configuration 1, 2, 3.1), it can be seen that the error of the theoretical result and the measured efficiency is within $\pm 0.4 \%$. This result demonstrates the validity of the energy utilization analysis in Section 2. It is obvious that the energy utilization efficiency is increased after dividing batteries into multiple groups. Considering the second scenario in the third configuration, the energy utilization efficiency is highest if batteries are sorted first before grouping. Due to the randomness of regression process, the capacity distribution of batteries inside each pack can be different as shown in configurations 3.1 and 3.2. Thus, the theoretical value under a certain confidence level represents a probability of the real energy utilization efficiency.

\subsection{Experiment 2}

Based on the proposed model and the preliminary verification in Experiment 1, a prototype has been designed and built to realize flexible groups. $\mathrm{LiFePO}_{4}$ batteries are retired from a company's EV with mean 49.46 Ah and a standard deviation 1.50 Ah. The number of total batteries used in the prototype is 112, and the voltage of the AC bus is $220 \mathrm{~V}$. Parameters of batteries and converters are used in the model, the resulting highest energy utilization efficiency is $89.4 \%(\alpha=0.95)$, where the 
number of groups is seven. Therefore, the configuration of the prototype is constructed accordingly, with seven groups of 16 batteries each.

Figure 11 shows a picture of the prototype in which basic power control has been implemented. The power factor is 1 in phase A as in Figure 12a. Figure 12b shows the battery current waveforms of each module in phase $\mathrm{B}$, where the DC component of current in each module of phase $\mathrm{B}$ is $2.883 \mathrm{~A}$, $4.535 \mathrm{~A}$, and 7.283 A, respectively. Individual power control is realized in the BESS, which shows the feasibility and flexibility of the prototype.

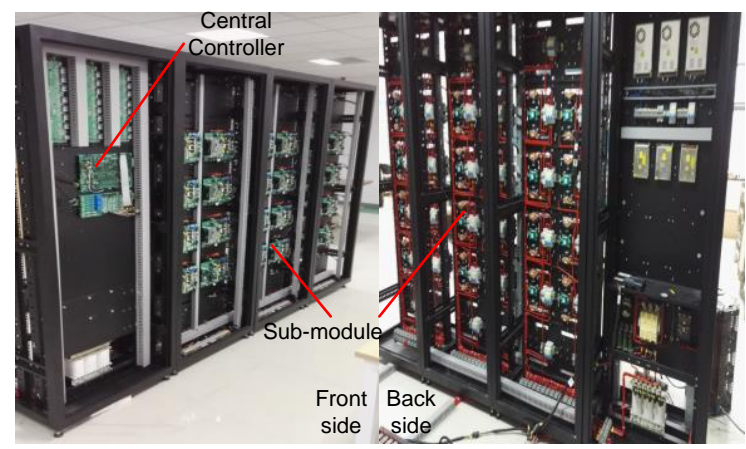

Figure 11. Picture of the prototype.

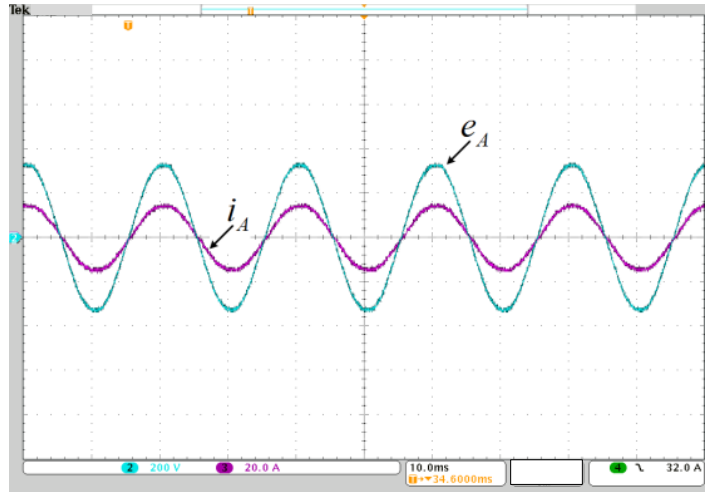

(a)

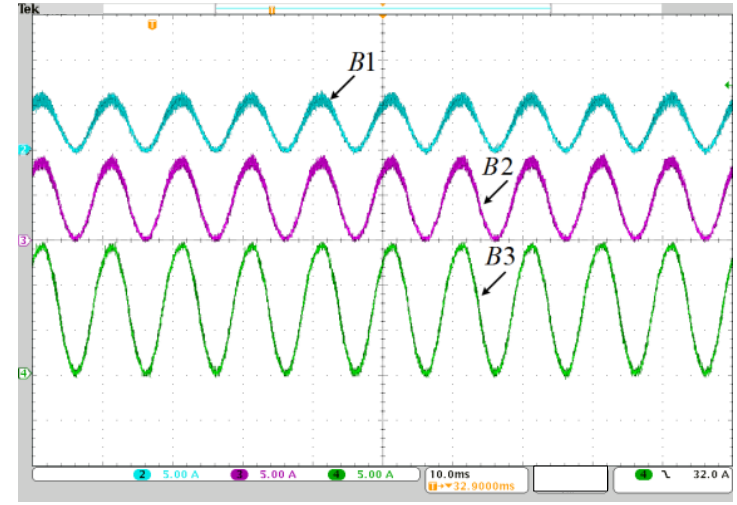

(b)

Figure 12. Waveforms of flexible groups during charge: (a) AC current and voltage in phase A; and (b) DC current of each module in phase B.

\section{Conclusions}

Flexible groups are used to solve capacity mismatches among a large scale of batteries by dividing them into groups through converters. The number of batteries in a pack, the capacity distribution characteristics, and the converter efficiency are studied comprehensively in the BESS. Energy utilization efficiency depends on the energy utilization of the battery pack and the converter efficiency. It is shown in this paper that under different capacity distribution characteristics, the optimal number of groups varies. This basic analysis provides an optimization method for energy utilization efficiency of a BESS throughout its life cycle. The experiments further verify the analysis results. In addition to the energy utilization analysis for the system, there are some other issues related to flexible groups on a system design level or on a control and topology level. Future research will focus on the impact of resistance distribution on the relationship between the number of groups and the energy utilization efficiency of the flexible group.

Acknowledgments: This work is financially supported by China National Energy Administration under Grant NY20150301. 
Author Contributions: In this paper, author contributions are as follows: Jiuchun Jiang conceived the main points of this paper. Weiping Diao, Jiuchun Jiang and Caiping Zhang conceived and designed the experiments; Hui Liang and Caiping Zhang, Yan Jiang performed the experiments; Weiping Diao analyzed the data; Yan Jiang, Leyi Wang and Biqiang Mu contributed reagents/materials/analysis tools; Weiping Diao wrote the paper.

Conflicts of Interest: The authors declare no conflict of interest.

\section{Abbreviations}

The following abbreviations are used in this manuscript:

BESS Battery Energy Storage System

DG Distribution Generation

ESS Energy Storage System

SOC State of Charge

DOD Depth of Discharge

BMS Battery Management System

$\mathrm{SOH}$ State of Health

\section{References}

1. Li, Y.; Han, Y. Power electronics integration on battery cells. In Proceedings of the Applied Power Electronics Conference and Exposition (APEC), 2014 Twenty-Ninth Annual IEEE, Fort Worth, TX, USA, 16-20 March 2014; pp. 3318-3322.

2. Wen, F. Study on Basic Issues of the Li-ion Battery Pack Management Technology for Pure Electric Vehicles; Beijing Jiaotong University: Beijing, China, 2009; pp. 8-9.

3. Soong, T.; Lehn, P. Assessment of Fault Tolerance in Modular Multilevel Converters with Integrated Energy Storage. IEEE Trans. Power Electron. 2016, 36, 4085-4095. [CrossRef]

4. Zhang, Z.; Cai, Y.Y.; Zhang, Y.; Gu, D.J.; Liu, Y.F. A Distributed Architecture Based on Microbank Modules with Self-Reconfiguration Control to Improve the Energy Efficiency in the Battery Energy Storage System. IEEE Trans. Power Electron. 2016, 31, 304-317. [CrossRef]

5. Rothgang, S.; Baumhöfer, T.; van Hoek, H.; Lange, T.; De Doncker, R.W.; Sauer, D.U. Modular battery design for reliable, flexible and multi-technology energy storage systems. Appl. Energy 2015, 137, 931-937. [CrossRef]

6. Mukherjee, N.; Strickland, D. Control of second-life hybrid battery energy storage system based on modular boost-multilevel buck converter. IEEE Trans. Ind. Electron. 2015, 62, 1034-1046. [CrossRef]

7. Young, C.M.; Chu, N.Y.; Chen, L.R.; Hsiao, Y.C.; Li, C.Z. A Single-Phase Multilevel Inverter with Battery Balancing. IEEE Trans. Ind. Electron. 2013, 60, 1972-1978. [CrossRef]

8. Su, G.H. Multilevel DC link inverter. IEEE Trans. Ind. Appl. 2005, 41, 848-854. [CrossRef]

9. Gupta, K.K.; Jain, S. A Novel Multilevel Inverter Based on Switched DC Sources. IEEE Trans. Ind. Electron. 2014, 61, 3269-3278. [CrossRef]

10. Babaei, E.; Laali, S.; Alilu, S. Cascaded Multilevel Inverter With Series Connection of Novel H-Bridge Basic Units. IEEE Trans. Ind. Electron. 2014, 61, 6664-6671. [CrossRef]

11. Maharjan, L.; Yamagishi, T.; Akagi, H.; Asakura, J. Fault-tolerant operation of a battery-energy-storage system based on a multilevel cascade PWM converter with star configuration. IEEE Trans. Power Electron. 2010, 25, 2386-2396. [CrossRef]

12. Maharjan, L.; Inoue, S.; Akagi, H.; Asakura, J. State-of-charge (SOC)-balancing control of a battery energy storage system based on a cascade PWM converter. IEEE Trans. Power Electron. 2009, 24, 1628-1636.

13. Zheng, Z.; Wang, K.; Xu, L.; Li, Y. A Hybrid Cascaded Multilevel Converter for Battery Energy Management Applied in Electric Vehicles. IEEE Trans. Power Electron. 2014, 29, 3537-3546. [CrossRef]

14. Vasiladiotis, M.; Rufer, A. Analysis and control of modular multilevel converters with integrated battery energy storage. IEEE Trans. Power Electron. 2015, 30, 163-175. [CrossRef]

15. Wang, L.Y.; Wang, C.; Yin, G.; Lin, F.; Polis, M.P.; Zhang, C.P.; Jiang, J.C. Balanced Control Strategies for Interconnected Heterogeneous Battery Systems. IEEE Trans. Sustain. Energy 2016, 7, 189-199. [CrossRef]

16. Arifujjaman, M. A comprehensive power loss, efficiency, reliability and cost calculation of a $1 \mathrm{MW} / 500$ kWh battery based energy storage system for frequency regulation application. Renewable Energy 2015, 74, 158-169. [CrossRef] 
17. Mukherjee, N.; Strickland, D.; Cross, A.; Hung, W. Reliability estimation of second life battery system power electronic topologies for grid frequency response applications. In Proceedings of the 6th IET International Conference on IET Power Electronics, Machines and Drives (PEMD 2012), Bristol, UK, 27-29 March 2012; pp. 1-6.

18. Zhang, C.P.; Jiang, J.C.; Zhang, W.G.; Wang, Y.K.; Sharkh, S.M.; Xiong, R. A novel data-driven fast capacity estimation of spent electric vehicle lithium-ion batteries. Energies 2014, 7, 8076-8094. [CrossRef]

19. Schuster, S.F.; Brand, M.J.; Berg, P.; Gleissenberger, M.; Jossen, A. Lithium-ion cell-to-cell variation during battery electric vehicle operation. J. Power Sources 2015, 297, 242-251. [CrossRef]

20. Zhang, C.; Jiang, J.; Zhang, W.; Wen, F. Determination of SOC of a battery pack used in pure electric vehicles. In Proceedings of the 2012 Asia-Pacific Power and Energy Engineering Conference, Shanghai, China, 27-29 March 2012; pp. 1-4.

21. Order Statistics. Available online: http://www.math.uah.edu/stat/sample/OrderStatistics.html (accessed on 3 March 2016).

22. The Central Limit Theorem. Available online: http://www.math.uah.edu/stat/sample/CLT.html (accessed on 3 March 2016).

23. Paul, S.; Diegelmann, C.; Kabza, H.; Tillmetz, W. Analysis of ageing inhomogeneities in lithium-ion battery systems. J. Power Sources 2013, 239, 642-650. [CrossRef]

24. Huang, B.X.; Chen, Y.S. Prediction of power losses in three-phase inverter based on system simulation. Micromotors 2009, 9, 49-52.

(C) 2016 by the authors; licensee MDPI, Basel, Switzerland. This article is an open access article distributed under the terms and conditions of the Creative Commons Attribution (CC-BY) license (http://creativecommons.org/licenses/by/4.0/). 